\title{
DE LAS CONFERENCIAS DE PARÍS AL PROYECTO DEL SISTEMA: LAS ÚLTIMAS PRESENTACIONES HUSSERLIANAS DE LA FENOMENOLOGÍA
}

Hernán Gabriel Inverso* hernaninverso@gmail.com

RESUMEN Husserl emprendió varias presentaciones sintéticas de la fenomenología con el propósito de darla a conocer ante públicos amplios. En el presente trabajo estudiaremos el enfoque de las Conferencias de París, su proyección en las Meditaciones cartesianas y el destino del proyecto de la versión alemana en tensión con la redacción del Sistema de filosofía fenomenológica, a los efectos de establecer sus rasgos distintivos, evaluar los alcances del abandono del neocartesianismo, que suele asociarse con el último período de producción de Husserl y, situar la dirección de estas últimas presentaciones en el clima intelectual que les dio origen.

Palabras clave: fenomenología, cartesianismo, trascendental, empatía.

Abstract: Husserl undertooks several synthetic presentations of phenomenology in order to make it known to broader audiences. In the present work we will study the approach of the Paris Lectures, their projection in the Cartesian Meditations and the idea of the German version in tension with the writing of the System of phenomenological philosophy, in order to establish its distinctive features, to evaluate the abandonment of neocartesianism that is usually associated with the last period of Husserl's production and to identify the direction of these presentations in the intellectual climate that gave rise to them.

Keywords: phenomenology, cartesianism, transcendental, empathy.

\footnotetext{
* Universidad de Buenos Aires. Artigo recebido em 24/01/2017 e aprovado em 26/04/2017.

1 Véase, por ejemplo, D. Moran $(2012,28)$.
} 
Por invitación del Institut d'études germaniques y de la Société francaise de Philosophie, a fines de febrero de 1929, Husserl dictó dos lecciones de dos horas en alemán en la Sorbona, en el Amphithéâtre Descartes. Algunos conectan esta actividad, así como otras orientadas a la publicidad de su filosofía fuera de los claustros de Friburgo con la necesidad de posicionar su obra frente a la estrella creciente de Heidegger, especialmente por los riesgos de que la cercanía vital y teórica entre ambos autores terminara aumentando el peso del discípulo hasta fagocitar el del maestro, como ha pasado muchas veces en la historia del pensamiento. ${ }^{1}$ Así fuese o no, lo cierto es que Husserl se acercó al círculo parisino y contó en su audiencia con Emmanuel Levinas, Lucien Lévy Bruhl, Jean Héring, Alexandre Koyré, Jan Patocka, Gabriel Marcel y Maurice Merleau-Ponty.

Su trabajo, que sería la base de las Meditaciones cartesianas, suele ser una de las puertas de ingreso a la filosofía de Husserl, sin contar con que se trata de una de las estrategias de presentación de la fenomenología y cuenta con versiones bien distintas. La idea de la fenomenología sugería en 1907 por primera vez la dimensión trascendental y establecía una mirada de neocartesianismo. Más tarde, en 1911, "La filosofía como ciencia estricta" situaba la fenomenología en el concierto de líneas adversarias. Ya en el período de Friburgo la Conferencia inaugural, pronunciada en 1917 al momento de asumir su cargo docente, ofrecía una síntesis general de aspectos centrales de sus aportes filosóficos hasta el momento. ${ }^{2}$ Un nuevo modelo de presentación se inaugura en 1922 en las Conferencias de Londres, las cuales forman el sustrato sobre el que más tarde, en 1929, Husserl delineó las Conferencias de París, el texto publicado como Meditaciones cartesianas y la conflictiva versión alemana de este, enlazado con el proyecto de retomar el Sistema de filosofía fenomenológica que constituía una idea desde el comienzo de la década del '20. Una vertiente alternativa, usualmente asociada con el abandono del cartesianismo, preanunciando las derivas de los textos de la Crisis, se plasma en el artículo para la Enciclopedia Británica de 1927 y en las Conferencias de Amsterdam de 1928.

En lo que sigue nos concentraremos en las presentaciones de la fenomenología del último período de producción de Husserl las cuales adoptan una impronta neocartesiana, se comenzará en primer lugar con las Conferencias de París, para señalar los puntos que les confieren identidad frente a las previas. En segundo lugar, consideraremos la versión de Meditaciones cartesianas, atendiendo a las variaciones de perspectiva que presenta respecto a las Confe-

1 *Universidad de Buenos Aires. Artigo recebido em 24/01/2017 e aprovado em 26/04/2017. Véase, por ejemplo, D. Moran $(2012,28)$.

2 Sobre la cuestión general de las presentaciones husserlianas de la fenomenología y en especial sobre los primeros textos de esta serie, véase $\mathrm{H}$. Inverso (2016a, 19-118). 
rencias de París, con la finalidad de evaluar la posición que en este momento adopta Husserl frente al cartesianismo y a la respuesta al riesgo del solipsismo que se liga con este tema. Esto nos permitirá advertir matices que muestran la ductilidad del pensamiento husserliano, lejos de algunos esquemas reductivos que malentienden algunos planteamientos respecto a la subjetividad y la intersubjetividad. Finalmente nos referiremos a los avatares que rodearon la redacción de la versión alemana y al proyecto alternativo en un clima de presiones crecientes que enfatizan la importancia que confirió Husserl a la tarea de presentación integral de la disciplina. Esto hará posible delinear el valor de las últimas presentaciones husserlianas de la fenomenología para la comprensión de la dirección general del enfoque.

\section{El filósofo que comienza en français}

Las Conferencias de París tienen un cuidado extremo por la transmisión, en tanto Husserl se propone clarificar los puntos principales del enfoque con el propósito de ampliar su impacto en la comunidad filosófica. Revisemos sintéticamente el rumbo del texto para componer el horizonte general. En la primera lección, a diferencia de la estrategia que utilizó en la experiencia de Londres, Husserl enfatiza la relación entre la presentación en Francia y la relación de la fenomenología con Descartes concebido como el gran pensador de esa patria, desdibujando la figura de Platón. ${ }^{3}$ El filósofo que comienza, sin embargo, como encarnación de la filosofía como unidad universal de las ciencias con fundamentación completa, sigue ahí, al igual que el acto de derrocamiento de todas las ciencias predadas, de nuevo en un marco de una autenticidad ética que replica la caracterización de lo ético-cognitivo de las Conferencias de Londres. ${ }^{4}$ El planteo cartesiano encarna "el prototipo de las meditaciones necesarias para todo filósofo que de nuevo comience", 5 en un inicio que es el de la filosofía y a la vez el de la filosofía primera. Se buscan, en efecto, vías apodícticas que permitan acceder desde la interioridad pura a la exterioridad objetiva en un proyecto orientado a lo eterno planteado con una fraseología teleológica: “No entrañará esta continua tendencia un sentido eterno, y para nosotros una gran tarea que nos es impuesta por la historia misma y en la que estamos todos llamados a colaborar?". ${ }^{\circ}$

Husserl pretende, por un lado, subrayar los elementos de continuidad con la inspiración cartesiana, aunque a la vez rechaza puntos centrales que han

3 Hua I, 3.

4 Véase Hua XXXV, 314. Sobre las Conferencias de Londres, véase R. Sánchez Soberano (2012) y H. Inverso (2016a, 80-99).

5 Hua l, 4.

6 Hua I, 5. 
hecho decir a P. Ricoeur que la radicalización no lleva a una renovación sino a una negación de las raíces cartesianas. ${ }^{7}$ El reemplazo de la duda cartesiana por la epoché fenomenológica y del ego empírico por un ego trascendental invitan a listar diferencias, tales como las que se ven en Street Fulton y más tarde en Gurwitsch, hasta que en 1970 Landgrebe refería un abandono del cartesianismo por exploración de nuevas temáticas que se consuman en la desaparición progresiva de la noción de filosofía primera, la cual apenas es mencionada en Meditaciones Cartesianas y, en la Crisis es directamente colocada entre paréntesis como muestra de la dilución de la meta de apodicticidad. ${ }^{8}$ Más tarde S. Geniusas revisó críticamente los estudios de Landgrebe para señalar aspectos endebles ligados especialmente con la presencia de temáticas supuestamente tardías en textos tempranos que no se ajustan al esquema de abandono progresivo, tal como en los casos de las nociones de horizonte y doble reducción. ${ }^{9} \mathrm{La}$ discusión sobre el problema de las vías a la reducción se apoya en este sustrato de abandono o persistencia del neocartesianismo, que para evitar malentendidos puede ser comprendido como la búsqueda de apodicticidad que haga de la fenomenología una verdadera filosofía que sostenga el edificio entero del conocimiento. Partiendo del contexto intelectual de fines del siglo XIX y con el propósito de confrontar con el naturalismo y el historicismo en boga, la figura de Descartes ofrece una base que habrá de ser renovada para servir a un nuevo entorno y hace falta, en todo caso, estudiar las torsiones que esta relación adopta en los distintos contextos.

En este sentido, la tarea orientada a lo eterno en contraste con el trabajo para el aquí y ahora que adviene en las Conferencias de París hace resonar los corolarios del artículo "La filosofía como ciencia rigurosa", pero suma ahora una suerte de adelanto de la línea persistente que cobraría más vigor en los últimos años de Husserl y se asocia con los textos de la Crisis. No se trata de una teleología pensada como narrativa de un progreso inevitable, al estilo hegeliano, ni mucho menos a un relato de decadencia de tipo nietzscheano o spengleriano, sino del resultado de una puesta entre paréntesis de los grandes relatos metafísicos, ya que tal propuesta fenomenológica se da en el marco de la suspensión de la tesis de efectividad y por lo tanto sin compromiso con la expresión de un ser que cargue con el imperativo teleológico. La teleología reposa, en todo caso, en una indicación de la razón y su desideratum, lo cual vale tanto para el destino de Europa como para el decurso de la fundamentación de las ciencias. ${ }^{10}$ 
Husserl se interna, entonces, en el ejercicio del filósofo que comienza buscando evidencia, y debe rápidamente descartar el conocimiento científico y también la experiencia del mundo, ya que necesitaría de una crítica de la experiencia sensible de la que no se dispone. Siguiendo a Descartes, se impone el giro hacia la subjetividad que revela el ego cogito como base apodíctica. Nada diferente de esto puede ser tomado como existente, pero todo permanece como fenómeno, incluyendo juicios y valoraciones. Este mecanismo de epoché constituye el modo por el cual se accede al "ego puro con la corriente pura de mis cogitationes". ${ }^{11}$ En rigor, Husserl caracteriza este punto como peligroso y sugiere que "es como si estuviéramos en la escarpada cresta de un risco y avanzar sobre ella con calma y seguridad decidiera sobre la vida filosófica y la muerte filosófica (Und doch ist es, als wären wir auf einem steilen Felsgrat, auf dem ruhig und sicher fortzuschreiten über philo- sophisches Leben und philosophischen Tod entscheidet)". ${ }^{12}$

El error cartesiano se da precisamente por apresuramiento, a pesar de haber entrevisto precisamente allí un gran riesgo, y ese apresuramiento está dictado por la escolástica que corre como sustrato del proyecto cartesiano sin poder ser expulsado. ${ }^{13}$ Es la metafísica tradicional, pero también el deslumbramiento por el modelo matemático lo que lo lleva a comparar el ego cogito con un axioma y a concebir el resto del programa como un desarrollo deductivo, sin advertir la trampa y la dilución del proyecto de fundamentación radical que se esconde en esta elección. ${ }^{14}$ La imagen que usa esta vez para ejemplificar el equívoco cartesiano es que habríamos "salvado un pequeño cabillo de mundo, que sería para el yo filosofante lo único incuestionable del mundo", y ahora se trata "de inferir el resto del mundo mediante conclusiones bien dirigidas conforme a los principios innatos en el ego". ${ }^{15}$ La conversión del ego en sustancia cogitans lleva al "absurdo realismo trascendental", ${ }^{16}$ y por eso pierde de vista la subjetividad trascendental. El corolario es que no hay mundo y por ende no hay ciencias positivas, ni actos psíquicos asociados con un yo con cuerpo y por tanto mundano, de modo que tampoco hay psicología empírica, y precisamente con esto se ha ganado el yo puro.

El segundo paso indica la salida del apresuramiento que lleva a arrojarse a la recuperación del ser, y sugiere proseguir, al contrario, hacia una fundamentación trascendental. Las lamentaciones por el extravío heideggeriano en

la concepción cartesiana del error, véase C. Wee (2006, 77-150) y E. Scribano $(2016,599-613)$ y sobre la persistencia de motivos escolásticos, R. Ariew $(1999,7,38)$ y L. Velasco Guzmán (2010). 
el terreno de la antropología en las que Husserl prorrumpirá más tarde son plenamente comprensibles a la luz de estos planteos, ${ }^{17}$ dado que constituirían una iteración de la precipitación cartesiana que entrevé el punto arquimédico, pero en su desesperación por volver al mundo y rehabilitar su conocimiento pierden la radicalidad necesaria para avanzar en la fundamentación.

Advierte entonces Husserl de este modo la esfera de ser nueva e infinita, aquella que en las Conferencias de Londres llamaba "proto-selva fenomenológica", que otorga la experiencia trascendental y con ello gesta una nueva ciencia. ${ }^{18}$ Esta nueva ciencia es egología pura, pensada como un paralelo de lo que la psicología empírica considera como experiencia interna. La intencionalidad aparece mencionada con el protocolo de las Conferencias de Londres, es decir, mediante la indicación de la necesidad de pasar a la formulación triple ego cogito cogitatum y, presenta una versión del mismo ejemplo que refuerza la relación entre los textos. ${ }^{19}$ Por esta vía traza los rasgos del polo objetivo y de los modos de la conciencia.

Cabe notar que en esta presentación se contrasta "el vago ego cogito" con el flujo de la vida cogitativa, para enfatizar la riqueza del campo que surge con la operación de reducción. Se dice que "Yo era trascendental también en cuanto yo que vivía naturalmente, pero no sabía nada acerca de ello (auch als natürlich lebendes Ich war ich transcendentales, aber ich wusste davon nicht)", ${ }^{20}$ lo cual implica que ahora, con la revelación del yo puro, adviene la perspectiva del espectador desinteresado que provee "el último punto de vista pensable de la experiencia y el conocimiento (denkbar letzten Erfahrungs und Erkenntnisstandpunkt)" ${ }^{21}$ En este contexto se refiere Husserl al desdoblamiento operado por la reducción que constituye la primera versión de los tres niveles de subjetividad el cual presentará luego en el artículo de la Enciclopedia Británica y en la Crisis. ${ }^{22}$

El mundo como fenómeno trae a cuento las estructuras de la temporalidad

17 Sobre el distanciamiento entre Husserl y Heidegger, véase L. Landgrebe (2004, 293), H. Gadamer (1963, 19), F. Hermann (1981) y (1990, 15-22) y J. Adrián (2005, 157-173) y (2011, 213-238).

18 Hua I, 36.

19 Véase Hua XXXV, 81. Husserl plantea allí que el reino de los hechos egológicos es tal que las intuiciones puras posibilitadas por el método muestran que la conciencia se dirige a objetos intencionales, conocidos en modos intencionales variados. El sentido de esta afirmación se explicita en ambos textos a través del ejemplo de una casa y de la percepción del color de alguna de sus partes. De este modo se abren multiplicidades infinitas de propiedades fenomenológicas puras, de la mano de las distinciones de orientación, la alteración de la perspectiva, la referencia a la aparición de la propia corporalidad vivida, en tanto mi cuerpo es un punto cero de orientación constante y participa de todas las cosas percibidas. presenta la vía psicológica, en la línea que lo hará más tarde la Crisis. Sobre la cuestión de las vías, véase H. Inverso (2016b). 
fenomenológica inherente al fenómeno mismo llevando a plantear la síntesis como rasgo fundamental de la conciencia, lo cual dicta la diferencia entre contenidos reales (reell) e ideales (ideell) de la conciencia, es decir, lo que existe en la conciencia y lo que la conciencia mienta, el objeto intencional, meramente intencional. ${ }^{23}$ La descripción está orientada a exponer la estructura que conforma el sentido, para lo cual avanza advirtiendo las tipologías que se dan en el flujo (percepción, recuerdo, conciencia retencional, etc.) y por tanto la posibilidad de explorar la estructura regular de la subjetividad trascendental. ${ }^{24}$

La cuestión de la estructura de la subjetividad trascendental adviene en la tercera conferencia, donde se presenta el ámbito de los problemas constitutivos, que refieren a la razón, la realidad, la conciencia y el ser verdadero. Los problemas constitutivos abarcan la fenomenología trascendental última, mientras considera la totalidad sistemática de las vivencias reales y posibles referibles al ego y sus síntesis posibles. Esto quiere decir que la constitución de un tipo objetual tiene que ver con el modo de darse evidente según el tipo de experiencia posible que le cabe. ${ }^{25}$ En este marco, el ser del ego es ser en constante autoconstitución y fundamento para toda constitución de lo trascendente. Es el polo estable de convicciones persistentes y es parte fundamental del método fenomenológico el descubrimiento de su a priori innato. ${ }^{26}$

A propósito de esto cabe señalar que muchas veces las simplificaciones llevan a acusar a la fenomenología de tener una identidad logocéntrica dominada por una intencionalidad objetivante y opresora. Muy por el contrario, si bien los aspectos constitutivos son centrales en algunos desarrollos, Husserl se encarga de aclarar repetidas veces la mayor complejidad del asunto, afirmando por ejemplo, en las Lecciones de psicología fenomenológica que el yo "no es un polo muerto de identidad", y que su dinamismo es mucho más rico. En este sentido, “es el yo de las afecciones y de las acciones. El yo que en la corriente de las vivencias sólo tiene su Vida en el hecho de que, por una parte, como intencional, produce intenciones, con ella y, por otra parte, porque siente estímulos por parte de esas objetividades, es rozado por ellas como sintiente, es atraído hacia ellas y motivado a la acción por parte de ellas". ${ }^{27}$

Sobre esta distinción, véase el tratamiento en La idea de la fenomenología (Hua II, 43).

27 Es ist das Ich der Affektionen und Aktionen, das Ich, das im Strom der Erlebnisse nur darum sein Leben hat, einerseits weil es in diesen, als intentionalen, Intentionen übt, also auf ihre intentionalen Gegenständlichkeiten gerichtet und mit ihnen beschäftig ist, und andererseits, weil es von diesen Gegenständlichkeiten Reize erfährt, von ihnen als fühlendes berührt wird, zi ihnen herangezogen, con ihnen Aktionen motiviert wird (Hua IX, 208-209). 
Este punto es de relevancia, dado que varias críticas parecen desdibujar la dimensión afectiva y sintiente del yo, así como el dispositivo por el que es punto de irradiación, pero también de incidencia de afecciones que lo atraen sobre la base de un protoyo ligado al fluir primigenio. ${ }^{28}$ Frente a los estímulos del mundo, el yo es un sujeto de la afección tanto como de la actividad. R. Walton resume el haz de elementos que se dan cita en el yo, diciendo: "el yo no sólo es 'punto de irradiación de actos', sino también 'punto de incidencia de afecciones'. Así, no sólo está involucrado en el nominativo de los actos, sino también en el acusativo del emplazamiento ético y en el dativo del ocurrirme algo". ${ }^{29}$ El yo se despliega en el arco de un entramado complejo que no se reduce al paradigma de los actos. En efecto, si reconocemos en el sujeto -y en primer plano- elementos de afectividad, buena parte de los argumentos sobre la incapacidad del yo trascendental, para operar la recepción de la donación que atraviesa la fenomenología francesa, se debilitan. ${ }^{30}$

Estos planteos llevaron a Husserl a hacer una evaluación de los logros alcanzados, afirmando que el programa para hacer accesible tanto la esfera trascendental como la esfera absoluta del ser y de la constitución es una tarea difícil y, sólo en los últimos años había conseguido aclarar y ordenar los problemas, entre los que se cuentan los de la génesis, y por tanto, se ha conformado una efectiva teoría del conocimiento que contrasta con la tradicional. Siguiendo la conexión temática que ya está presente en las lecciones de Londres, se procede a una distinción entre los modos en que se ha enfocado el problema de la trascendencia, lo cual para la fenomenología implica la pregunta de cómo puede alcanzar significación objetiva lo que transcurre en la inmanencia de la conciencia, y en ese sentido cómo la evidencia puede ser más que un carácter de conciencia, todo esto sin recurrir, como Descartes, a la veracidad divina. ${ }^{31}$ Desde la fenomenología, por cierto, la respuesta es que todo el planteo es un contrasentido provocado por el error cartesiano, como hemos visto, y la actitud de la tradición posterior que sólo empeoró las cosas, sin ver que "la trascendencia es un carácter de ser inmanente, que se constituye en el interior del ego (Transzendenz iste in immanenter, innerhalb des ego sich konstituierender Seinscharakter)", ${ }^{32}$ y un fuera de la conciencia es absurdo. La auto-exposición pura del yo implica un idealismo trascendental, pero de sentido nuevo, que no

Hua XV, 586. En efecto, así como hay un protoyo hay también una protogeneratividad asociada con los impulsos intersubjetivos que trazan los nexos vinculares de las generaciones (Hua XV, 178).

29 R. Walton $(2001,48)$

30 Sobre esta cuestión, véanse, por ejemplo, las críticas de M. Henry y J.-L. Marion y una posible respuesta a partir de la tematización fenomenológica de la excedencia y lo inaparente en H. Inverso (2016a, 229-287).

31 Hua I, 32.

32 Hua I, 32. 
se dedica a batallar con realismos, sino que expone el sentido que concierne a la trascendencia por el descubrimiento de la intencionalidad constituyente.

Con la explicitación de este programa, resta responder a la objeción del solipsismo, punto que, igual que en las lecciones de Londres, Husserl acomete de modo muy sintético. Precisamente en este punto adviene la mayor diferencia entre las Conferencias de París y Meditaciones Cartesianas, dado que en la revisión del texto el tratamiento sobre la empatía es lo que Husserl encuentra menos logrado, y emprende entonces, la redacción de una quinta meditación dedicada íntegramente a esta cuestión mientras lleva a cabo las correcciones. ${ }^{33}$

El tramo final, que es el mismo que conforma el final de Meditaciones Cartesianas integrado como $\S 64$, oficia de conclusión general. Husserl vuelve al tema de las ciencias y la necesidad de que se refieran a la fenomenología, ya que es en su carácter apriórico y trascendental de donde surge la posibilidad de una fundamentación última y conforma una ontología universal concreta que lleva de la egología a la fenomenología intersubjetiva, de ahí a las ciencias aprióricas y por último a las ciencias de hechos que quedarían comprendidas en la totalidad y en este movimiento alcanzarían también ellas fundamento. ${ }^{34}$ Este proyecto sustituye a las variantes metafísicas ingenuas y muestra el despliegue en el que "dentro de la esfera monádica fáctica, y en cuanto posibilidad esencial ideal en toda esfera monádica concebible, se presentan todos los problemas de la facticidad contingente de la muerte, del destino, de la posibilidad de una vida subjetiva y comunitaria reivindicada, en un sentido particular, como plena de sentido, por ende también los problemas del sentido de la historia, etc.". ${ }^{35}$

La lista apunta a incluir explícitamente como temática de esta filosofía universal todo el ámbito práctico y muy especialmente el espectro de fenómenos a los que se dirigen los estratos de la generatividad y la inapariencia, que junto con la estaticidad y la geneticidad completan el arco de atención a la fenomenicidad. ${ }^{36}$ No hay, entonces, aspectos ajenos a la perspectiva fe-

La estructura no es novedosa y plantea muy brevemente que si hemos hablado de una egología y el yo es una mónada, aun así no hay solipsismo, porque el yo puro experimenta a los otros realmente, es decir, que los experimenta en el plexo del mundo, pero con una peculiaridad que amerita un tratamiento especial, dado que se los experimenta al experimentar al mundo y a sí mismo. En este sentido, hay una respuesta al problema de las otras mentes, y para dar cuenta de esto se apela a la empatía, que implica una "apercepción de similitud", a la manera en que Leibniz afirmaba que en mi mónada se reflejan mónadas ajenas. Así, el ego trascendental pone necesariamente un alter ego trascendental y con ello emerge el plano de la intersubjetividad (Hua I, 35). Esto hace de la fenomenología una monadología fenomenológico-trascendental. Véase sobre este punto, R. Walton (2001), R. Bernet, I. Kern y E. Marbach (1995, 305-317), D. Zahavi (2005, 359-375). Hua I, 38.

Aber innerhalb der faktischen monadischen Sphäre, und als ideale Wesensmöglichkei in jeder erdenklichen, treten alle die Probleme der zufälligen Faktizität, des Todes, des Schicksals, der in einem besonderen Sinne als sinnvoll geforderten Möglichkeit einzelsubjektiven und gemeinschaftlichen Lebens auf, also auch sagen: es sind die ethischreligiösen Probleme des Sinnes der Geschichte, usw. (Hua I, 39). 
nomenológica y todos conforman un sistema integrado. ${ }^{37}$ La condición es la correcta aplicación del método, que lleva al autoconocimiento, rasgo que lo lleva a afirmar que: "La sentencia délfica gnôthi seutón ha cobrado un nuevo significado. La ciencia positiva es ciencia absorta en el mundo. Hay que perder primero el mundo por medio de la epoché para después recuperarlo en el autoexamen universal. Noli foras ire, dice San Agustín, in te redi, in interiore homine habitat veritas". 38

No son fórmulas cartesianas sino voces venidas de la antigüedad las que acompañan el cierre de las Conferencias. Por un lado la voz délfica, pero a la vez socrática, que recupera de este modo un lugar para la tradición platónica que había quedado fuera de consideración, después de habérsele concedido suma importancia en la reconstrucción historiográfica de las Conferencias de Londres. ${ }^{39}$ "Conócete a ti mismo" resulta en este contexto una invitación a la reflectio y con ello a la posibilidad de internarse en los enormes espacios que animan el mundo fenomenológico. Por otro lado, Agustín trae los sonidos del medioevo, que habían sido censurados bajo la categoría de escolástica que contaminó y debilitó el impulso de Descartes, pero vuelve ahora con la fuerza de admonición para decir en una variación del De vera religione, XXXIX.72: "no quieras ir fuera; vuelve a ti mismo; en el hombre interior habita la verdad". En efecto, las ciencias están absortas en el mundo, en tanto no salen de la actitud natural, con el efecto de perder toda oportunidad de fundamentación. Para ello es preciso perder el mundo, es decir, es preciso ponerlo entre paréntesis para recuperarlo luego, no por el recurso de la deducción, sino por el poder de la reflectio fenomenológica y la prosecución del método. Las Conferencias de París recorren, así, una serie de puntos nodales que enfatizan la importancia de la elección de los dispositivos básicos como clave para una investigación radical, aunque, por supuesto, no agotan los puntos problemáticos, y esta cuestión tiñe, como veremos, las reelaboraciones posteriores que se desprenden de este intento.

\section{Las Meditaciones Cartesianas y la pervivencia de lo momentáneo}

La intención sostenida de ofrecer una visión introductoria de conjunto a la fenomenología llevó a Husserl en 1929 a preparar el texto de las lecciones

hemos dedicado en H. Inverso (2016a), (2016b) y (2017), siguiendo los lineamientos abiertos por A. Steinbock (1995), (1998) y (2003) respecto de la generatividad.

37 Nótese al pasar, hasta qué punto los problemas de fundamentación de las ciencias del espíritu que desvelaban a Dilthey quedan en esta perspectiva integrados en el programa general sin necesidad de establecer diferencias tajantes con el resto de las disciplinas.

Das delphische Wort: gnôthi seautón hat eine neue Nedeutung gewohnen. Positive Wissenschaft ist Wissenschaft in der Weltverlorenheit. Man muss erst die Welt durch Epoché verlieren, um sie in universaler Selbstbesinnung wiederzugewinnen. Noli foras ire, sagt Augustinus, in te redi, in interiore homine habitat veritas (Hua I, 39). 
de París como publicación, en lo que serían las Meditaciones cartesianas, en una traducción francesa realizada por E. Lévinas y G. Pfeiffer. Meditaciones cartesianas no es una versión definitiva, dado que Husserl continuó trabajando en la versión alemana en virtud de que la versión francesa no lo convencía. Estas dudas se extendieron a los críticos, para verificar esto, basta con revisar la introducción de A. Smith a la traducción inglesa de 2003, donde afirma que se trata de un texto imperfecto: la brevedad conspira contra la claridad hasta el punto de que resulta ininteligible para quien no cuenta ya con conocimientos sobre fenomenología, lo cual lo hace inútil como introducción.

Sin embargo, esta obra es por lejos una de las más conocidas de Husserl y es usual buscar en ella una introducción general, a pesar de las dificultades que plantea. En efecto, es una versión ampliada, pero también complejizada de las Conferencias de París a las que acabamos de referirnos, por tal motivo mencionaremos aquí dos puntos en los que se produce una toma de distancia y que a menudo se extrapolan como indicación de líneas de fondo del pensamiento husserliano de un modo que simplifica en extremo procesos complejos. El punto más controvertido tiene que ver con el abandono de la vía cartesiana, que convive con la vía psicológica en la década de 1920 y luego pierde vigor en la Crisis. Son conocidos los pasajes contrastantes de esta obra que siembran dudas sobre el cartesianismo, ${ }^{40}$ apelan al mundo de la vida y lo predado, ${ }^{41}$ a la psicología y a la doble reducción fenomenológica y trascendental, ${ }^{42}$ dando lugar a un escenario de vías en conflicto. Precisamente la actitud crítica respecto de la vía cartesiana ha llevado a sostener a algunos intérpretes que no puede tratarse de una tesis que Husserl sostuviera seriamente en la época de Conferencias de París y fue sólo por el hecho de estar en la patria de Descartes que obtuvo allí un papel central. ${ }^{43}$ La situación es claramente más compleja y, en rigor, la adopción de una vía cartesiana, psicológica u ontológica, con el objetivo de seguir la caracterización tradicional de I. Kern que responde a los pasajes de la Crisis que mencionamos y también al tipo de fenómenos estudiados y no a una opción que requiera el abandono irremisible del resto. ${ }^{44}$ Aunque no nos detendremos aquí en este punto, interesa puntualizar que la persistencia del tópico del acceso por vía cartesiana al plano de lo trascendental en las últimas presentaciones complica en extremo las respuestas simplistas que pretenden interpretar el pensamiento husserliano en una clave de alejamiento progresivo del cartesianismo. ${ }^{45}$

41 Hua VI, 159. 
En efecto, la serie de autopresentaciones tempranas adhiere a parámetros neo-cartesianos estableciendo las críticas y distanciamientos que caracterizan al modelo husserliano, y esta línea se mantiene vigente también en las últimas presentaciones según vimos, lo cual permite ordenar brevemente los vínculos y diferencias que esta asociación supone de un modo aplicable en general al pensamiento de Husserl. Notemos tres vínculos principales que se mantienen constantes y dan por tierra con la idea de un abandono sin más del cartesianismo: en primer lugar, la propuesta de duda metódica que descarta todas las creencias inspira la idea de puesta entre paréntesis necesaria para salir de la actitud natural; en segundo lugar, el cogito pone el foco en la subjetividad contra los criterios externos, idea que Husserl profundiza en su estudio de la intencionalidad y; en tercer lugar, ambas filosofías comparten un proyecto de filosofía como ciencia rigurosa, el cual Descartes veía en el modelo matemático y Husserl despliega en su idea de coordinación de las ciencias al abrigo de la fundamentación fenomenológica.

También en las diferencias encontramos una línea de crítica persistente que no se transforma ni tiende al crecimiento. En primer lugar, la idea de que el ego es el principio en una cadena deductiva, a la manera de las matemáticas, es incompatible con el enfoque husserliano, el cual se preocupa por la experiencia pura e irreductible a un modelo formal de ese tipo; en segundo lugar, Husserl considera un extravío cartesiano la sustancialización del ego, que lo torna un objeto del mundo y por ende algo extraño a la subjetividad pura, así como la orientación hacia la res extensa que crea a juicio de Husserl un dualismo altamente problemático; en tercer lugar, en el terreno del método Husserl confiere una importancia fundamental a dos puntos que no tienen lugar en el planteo cartesiano: la salida de la actitud natural por vía de la reducción y la estructura intencional que hace que un acto tenga tres elementos cristalizados en la fórmula ego cogito cogitatum. La convivencia de esta tensión que atraviesa toda la obra de Husserl señala hacia un diagnóstico estable que reconoce logros, identifica equívocos peligrosos y deja de lado la idea de un alejamiento progresivo.

Un segundo punto problemático atañe a la quinta meditación que, como dijimos, es la mayor novedad de Meditaciones cartesianas. ${ }^{46}$ Entre los puntos principales del tratamiento de la intersubjetividad monadológica cabe notar que la quinta meditación trata de la empatía en tanto tipo de operación, distinta de la percepción y la reflexión, dirigida a los alter ego, por la cual se presentan a la experiencia, objetos que son a la vez sujetos y hacen del mundo ya no un reservorio de datos para una conciencia solitaria sino, un ámbito público atravesado por sentidos construidos de modo intersubjetivo. Husserl escapa al planteo moderno conocido como "problema de las otras mentes", dado que no 
pretende probar la existencia de otros sujetos, sino explicar cómo llegamos a experimentar "lo otro" saliendo más allá de nuestro mundo primordial. ${ }^{47}$

La constitución del mundo objetivo es "el paso que nos lleva al 'otro' yo", ${ }^{48}$ con la reducción como requisito, de modo tal que el yo suspende "todos los efectos constitucionales de la intencionalidad relacionada inmediata o mediamente con otra subjetividad", y eso provoca una "reducción a mi esfera trascendental de peculiar propiedad o a mi trascendental concreto yo mismo". ${ }^{49}$ Por esta vía se instala la esfera egológica, de modo que el ego soy yo mismo en mi primordial mismidad como unidad psicofísica en relación con el mundo primordial circundante. ${ }^{50}$ Mi propio yo es dador de sentido y centro de orientación, y produce la apertura al alter ego, una intencionalidad mediada por la presentación de su cuerpo material. En tanto tengo primariamente experiencia de mi cuerpo propio (Leib), constituyo la experiencia del cuerpo físico (Körper), y, de manera inversa, accedo a los otros por su dimensión física objetual (Körper) y luego capto en su comportamiento como cuerpos físicos la dimensión de Leib que debe haber en cada uno. Así es que la "transferencia analogizante" lleva de la similitud en el cuerpo propio a la similitud en el ámbito de la conciencia, en el sentido de que reconozco conciencia también en los otros. ${ }^{51}$

Si aprehendo el cuerpo externo como un organismo animado, debo haber "derivado este sentido por medio de una transferencia aperceptiva a partir de mi organismo animado". ${ }^{52}$ La base motivacional para la "aprehensión 'analogizante" de ese cuerpo como otro organismo animado es la similitud con mi esfera primordial. ${ }^{53}$ Captar los alter ego implica una apercepción implenificable por medio de otras percepciones, y solamente es posible lograrlo con presentificaciones a través de la imaginación, pues ningún cambio de perspectiva da acceso directo a la conciencia ajena. ${ }^{54}$ Es la operación de empatía lo que construye la intersubjetividad, mientras que la reducción a la esfera de lo propio es un recurso metodológico y no un compromiso con el solipsismo. A esto se suma que la transferencia analogizante no es una inferencia a la manera de las aproximaciones inductivas al problema de las otras mentes. ${ }^{55} \mathrm{Al}$ contrario,

Hua I, 137-8. Sobre el problema de las otras mentes y su origen en la antigüedad, que refleja mejor el tipo de preocupación husserliana que sus versiones modernas, véase nuestro tratamiento en H. Inverso (2015, 21-39).

Sobre la empatía, véase R. Walton (2001).

Hua I, 140.

Hua I, 140-41.

Hua I, 150.

Sobre los puntos de contacto del argumento husserliano y el presentado por B. Russell para solucionar inductivamente el problema de las otras mentes en (1948:483 ss.), véase M. Braund (2012:10-11). 
constituye un proceso intencional que por apercepción hace aparecer una cosa en mi esfera primordial captada como cuerpo por su similitud con el cuerpo propio. Para dejar esto en claro Husserl afirma que "la apercepción no es una inferencia, no es un acto de pensamiento". ${ }^{56}$

Es claro que no sólo un mecanismo inferencial es innecesario, sino que contradiría todas las críticas que Husserl había señalado frente al cartesianismo precisamente por hacer depender la evidencia de recursos deductivos. La inferencia viciaría entonces, nuestro acceso primordial a los otros, por lo cual Husserl sostiene que no hay diferencia entre la transferencia analogizante que abre la experiencia de los otros y la experiencia cotidiana que refiere siempre a "una institución primaria en la cual un objeto con un sentido similar resultó constituido por primera vez". ${ }^{57}$

Las perspectivas husserlianas sobre el cuerpo señalan momentos de constitución que parten de la identificación de un punto original de orientación y luego de la dimensión cinestésica asociada con el sentido de "yo puedo", el cual lleva a la constitución del cuerpo como sentiente. Sobre la base de este proceso de constitución se despliega la parificación que responde, como síntesis pasiva, a la captación de una similitud entre mi cuerpo y el cuerpo percibido de los otros. ${ }^{58}$ Por esta vía se pasa de la esfera de lo propio a la interacción con otros que poseen sus propias esferas primordiales, lo cual conforma el punto en que la epoché temática que estableció un solipsismo instrumental deja paso al despliegue de una fenomenología comprometida con lo intersubjetivo. ${ }^{59} \mathrm{De}$ este modo queda establecida "la implicación intersubjetiva, la mezcla o imbricación (Ineinander) de los otros en la subjetividad trascendental que Husserl fundamenta explícitamente en la corporalidad". ${ }^{60}$

El ego trascendental como conciencia encarnada está enlazado con los otros y forma parte de una comunidad trascendental que tiene un mundo que es tal para este ensamble complejo de mónadas. Zahavi nota bien cuántos puntos de contacto existen entre estos planteos y los que desarrollará Merleau-Ponty, quien avanzará por los caminos abiertos por desarrollos como por ejemplo, la tercera parte de Sobre la fenomenología de la intersubjetividad, texto de la última época de Husserl donde considera que hablar de 'yo' es un equívoco propio de los primeros estadios de la investigación fenomenológica que muestra finalmente que el yo lleva en sí otros yoes", todos ellos atravesados por su 
dimensión de corporalidad. ${ }^{61}$ Esta redefinición que pone al cuerpo en primer plano abrirá las discusiones que emergen en las líneas herederas e inspiradas en la fenomenología husserliana que se verán cautivadas especialmente por los planteos de la conciencia encarnada y en interacción extrema con el mundo circundante. Así veremos multiplicarse las preocupaciones del último Husserl por actividades como la alimentación, el deseo y el acto sexual asociadas con la dimensión del Leib, lejos, a primera vista, de la conciencia que en el planteo de Ideas I no necesitaba un cuerpo.

En efecto, esas preocupaciones crecientes marcan el último período de producción husserliana y crecen en manos de sus continuadores. Sólo como efecto de los avatares de transmisión de la obra íntegra, debido a la publicación póstuma y tardía de muchos trabajos fundamentales, resulta comprensible la visión tradicional que asocia a Husserl con un neocartesianismo rígido y, por tanto, ve a sus continuadores como autores dispuestos a la ruptura con los elementos básicos del planteo fenomenológico primigenio. En esta línea de lectura la presencia de Descartes condiciona el planteo y fraseología con que se presenta el problema, cuando en rigor, el rechazo del procedimiento de prueba mediante deducción aleja el planteo del cartesianismo y lo redefine. No obstante, de modo más básico, D. Carr sugiere que en otros textos el acceso al alter ego no se plantea como una respuesta al solipsismo, sino únicamente como peculiaridad del tipo de objetos que son sujetos, no Gegenstände sino Gegen-Subjekte. ${ }^{62}$ En este sentido, la salida del solipsismo nunca implica la afirmación de existencia, dado que la reducción permanece activa. La objeción en general parece exagerar la adopción del planteo cartesiano, que, según muestra nuestro recorrido, es crítica desde el origen y deslinda claramente cuál es la inspiración que se adopta y cuáles las derivas que hay que corregir. ${ }^{63}$

\section{La 'moda pasajera' de una filosofía de la existencia}

Avancemos en el terreno más oscuro que se proyecta a partir de este modelo de presentación. Tras la publicación del texto francés, Husserl mantiene

61 Véase Hua XV, 586. Sobre este punto, véase además E. García (2012:58).

62 D. Carr (1987, 45-67).

63 El mayor problema que se identifica en esta línea es que Husserl llega a abogar por la igualdad de dignidad de egología y fenomenología intersubjetiva, lo cual es conflictivo, ya que si el rigor implica donación apodíctica, sólo lo egológico y su clima de solipsismo puede ser riguroso, y, al contrario, si hay igualdad, la donación apodíctica deja de ser un parámetro, dado que hemos visto que sólo se llega a lo intersubjetivo por transferencia analogizante. No trataremos aquí del problema de las vías, que provee más elementos para evaluar este punto, pero cabe mencionarlo aquí porque las interpretaciones encontradas son el producto de las variaciones efectivas que hay entre las presentaciones de esta época, las cuales debemos interpretar junto con las estrategias bien distintas del artículo de la Enciclopedia Británica y las Conferencias de Amsterdam que completan el arco de presentaciones. Véase T. Sheehan y R. Palmer (1997) y H. Inverso (2017), donde se discuten las líneas interpretativas a propósito de este punto. 
la intención de mejorarlo en función de la publicación de una versión alemana en el Jahrbuch für Philosophie und phänomenologische Forschung, según comenta a Roman Ingarden en una carta de febrero de $1931 .{ }^{64}$ Eugen Fink colabora en esta tarea, no sólo revisando el texto ya producido, sino redactando una sexta meditación que seguiría al conjunto de la revisión. Comenta Fink en una carta a H. Van Breda en 1946, en medio de los planes de publicación de la versión alemana: "Las buenas noticias acerca del trabajo de los Archivos Husserl y de sus próximas publicaciones es muy bienvenido. Sobre todo la edición del texto alemán de las Méditations Cartésiennes se necesita con urgencia. Como tal vez sabe, Husserl no estaba listo para publicar el texto alemán, porque vio limitaciones mayores en su presentación. En los años 1932-33, cuando Husserl estaba preocupado con la idea de este proyecto, producir una edición alemana, tuve que bosquejar para él un rediseño de las Meditaciones Cartesianas. [...] Después de la aparición del texto alemán, tal vez publique estas mis propuestas de revisión para las Meditaciones Cartesianas, propuestas que Husserl había aceptado. [...] Husserl había adoptado mis propuestas tan completamente que, para ese tiempo, me propuso que mis revisiones de su texto y mi enteramente nueva "Sexta meditación" (sobre la idea de una teoría trascendental del método) sea incluida en la edición alemana planeada y publicada bajo autoría compartida. Los desarrollos políticos después de 1933 hicieron imposible proceder con este plan." 65

La revisión para la publicación francesa había llevado dos meses que bastaron para dejar al descubierto inconvenientes y el incentivo de una corrección para una versión en alemán, lo que en algún sentido implicaba mayores responsabilidades en tanto involucraba una presentación general en el ámbito en que había surgido y evolucionado la teoría. La presión adicional suele asociarse con la figura de Heidegger, ${ }^{66}$ que impacta de varias maneras en Husserl. Algo así efectivamente se entrevé en las palabras del propio Husserl cuando le escribe a Ingarden: "El pequeño texto francés que va a aparecer en Pascua (unas cien páginas) no será una mera traducción del alemán, porque para el público alemán -en su presente situación (la moda pasajera de una filosofía de la "existencia", el abandono de la "filosofía como ciencia rigurosa")- lo que se necesita es una exposición más extensa y de mayor elaboración que convenga a la más alta problemática "metafísica", ${ }^{67}$

La idea de ampliar el texto se desdibujó ante la creciente sensación de que el enfoque íntegro tenía una estructura que invitaba al error. Se ha asociado el

64 HuDo III/III, 273.

65 Carta a Van Breda del 26/10/1946 (Fink Nachlass).

66 R. Bruzina (1995, X).

67 HuDo III/III, 262. 
cambio de planes con la lectura del texto de Misch, Filosofía de la vida y Fenomenología. Por cierto, Misch retomaba la posición de Husserl, pero presentaba una serie de malentendidos que hacían de Husserl un cultor del intelectualismo logicista, ajeno y prácticamente en las antípodas del espíritu de Dilthey, mucho mejor representado en los análisis heideggerianos. Hemos visto que, dejando incluso de lado las deferencias por Dilthey que manifiesta en la carta que le dirige en junio de 1911, Husserl creía que había entre ambos enfoques muchos puntos de contacto, que se habían multiplicado con los trabajos de Husserl de los últimos años, lo cual por sí solo justificaba la preocupación por una interpretación tan alejada de lo que pretendía proyectar. Obviamente hay que sumar que estas limitaciones terminaban dando una imagen de conjunto que dejaba la empresa husserliana por debajo de la heideggeriana, esa pasajera moda existencialista. A partir de ahí Husserl trabajó con Fink en el diseño de un Sistema de filosofía fenomenológica que tuviera todo lo necesario. Al trabajo de Fink se debe el texto introductorio sobre el mundo como lo predado a toda reflexión filosófica, el cual Husserl supervisó, y entre 1930 y 1931 desarrolló él mismo haciendo varios escritos sobre temas relacionados con el mundo de la vida y la intersubjetividad, ahora comprendida, a diferencia del enfoque en las Meditaciones cartesianas, con primordialidad junto con la mónada yoica. ${ }^{68}$

El peso del emprendimiento hizo que el trabajo no estuviera en un formato publicable con la premura requerida, por lo cual para este tiempo Husserl volvió a considerar la reescritura de las Meditaciones cartesianas, que encomendó a Fink. El nuevo proyecto implicaba ampliaciones y el agregado de dos meditaciones adicionales: la sexta sobre una crítica de la experiencia y la cognición fenomenológica y la séptima sobre el futuro de la metafísica de la fenomenología. Los proyectos de la versión alemana y el sistema continúan en pugna y Husserl oscila de uno a otro en sus prioridades, buscando sin descanso el modo de hacer conocer su filosofía, en un ánimo que califica de trágico en una carta a G. Albrecht: "Es una situación trágica, en la que, a pesar de que estoy absolutamente seguro de que en la última década llevé mi filosofía fenomenológica a la madurez, a la claridad y pureza, a una amplitud de problemas y métodos abarcativos que trazan el genuino sentido y la vía para la filosofía de todo el futuro, vino una nueva generación a escena que malinterpreta mis fragmentos y comienzos incompletos publicados en su sentido más profundo, que propaga una fenomenología supuestamente mejorada y me reverencia como a un viejo pasado. Así es que estoy otra vez filosóficamente solo, como estaba cuando empecé". ${ }^{69}$ 
Esta sensación acucia a Husserl en el último período y no se priva de relatarla repetidamente a sus conocidos, hasta que finalmente 1932 lo vio sumido en un período de depresión. Mientras tanto Fink había avanzado en la revisión y compuso la sexta meditación, de un modo tan renovado que introducía modificaciones que a juicio de Husserl trastornaban el texto, por lo cual la revisión se extendió bastante tiempo y llevó a Fink a redactar un prólogo en el que señalaba diferencias entre su pensamiento y el de Husserl. Este clima los sorprendió el verano de 1933 y el ascenso del nazismo, que pronto llegó a la Universidad y dictó la expulsión de todos los miembros judíos. Husserl se encontraba ya retirado y poco después fue alcanzado por la excepción para quienes hubieran perdido hijos en la Primera Guerra, pero simbólicamente fue afectado de manera directa, a lo cual se sumó el despido de su hijo Gerhart de la Universidad de Kiel. La responsabilidad de Heidegger como rector de la Universidad de Friburgo desde abril seguramente no ayudó al estado de ánimo de Husserl, quien se lamentaba de que "lo convulsionado de las épocas" no lo dejaban concentrarse y la sensación de impotencia frente a los hechos políticos del momento lo paralizaban. ${ }^{70}$

Fink, por su parte, sufrió la negativa de defender su tesis de Habilitación, que según los planes sería el texto de la sexta meditación. Los tiempos que siguieron no fueron más auspiciosos a pesar del trabajo sostenido y la inquietud creciente, hasta que finalmente en 1934 condujo a los textos de la Crisis a propósito de su participación en el Congreso Internacional de Filosofía de Praga sobre la presente tarea de la fenomenología. Esta irrupción vino de la mano de un corte de nudo gordiano respecto de los proyectos previos, los cuales quedaron de nuevo pospuestos en virtud de la sistematización de los manuscritos, en los que colaboraron Fink y Langrebe. ${ }^{71}$

Husserl comentó a D. Cairns en 1931 que había pensado erróneamente que componer una introducción a la fenomenología para una audiencia amplia era algo simple, pero luego vio que sus intentos de los últimos diez años, en el arco que va desde las lecciones de Londres a las de Paris y sus rediseños, carecieron todos de resultados satisfactorios. ${ }^{72}$ Husserl nunca concluyó el proyecto de la versión alemana, al que se refirió en 1930 como "el trabajo principal de mi vida", ${ }^{73}$ pero sabemos que no mantuvo las energías en ese proyecto y, por el contrario, se orientó hacia los trabajos también inconclusos que conformaron luego el texto de la Crisis.

Sinn und die notwendigen Wege vorzeichnet -eine neue Generation aufgetreten ist, die, meine veröffentlichten Bruchstücke und unvollkommenen Anfänge ihrem tiefsten Sinn nach missdeutend, eine vermeintlich verbesserte Phänomenologie propagiert und mich als den alten Papa verehrt, der nunmehr überholt sei. So bien ich wieder philosophisch einsam wie in meinen Anfängen (HuDo III/IX, 75).

70 HuDo, III/IX, 86-87.

71 HuDo I, 458.

72 D. Cairns $(1976,27)$.

73 K. Schumann $(1977,361)$. 


\section{Corolarios}

Si tenemos en cuenta, por un lado, las cuatro presentaciones que apelan al neocartesianismo conformadas por las Lecciones de Londres, las Lecciones de París, Meditaciones cartesianas y su versión alemana, por otro consideramos la emergencia de las estrategias asociadas con el artículo de la Enciclopedia y la Conferencia de Amsterdam, que parecen una suerte de vía suspendida, y finalmente observamos las derivas de la Crisis, asistimos al último período de Husserl como una suerte de sumatoria de proyectos superpuestos y en pugna por la atención del autor en el curso de una vida anunciando la finitud. Estos proyectos superpuestos no deben ser comprendidos como la proyección de una modificación metodológica asociada con el acceso al plano de lo trascendental, ya que si bien, en el primer grupo predomina la adopción de una vía cartesiana, en el segundo la vía psicológica y en el último adviene el planteo acerca del acceso a través del mundo de la vida. Este punto no resume el problema mucho mayor que se enraíza en la estrategia para dar cuenta de manera sintética de la fenomenología íntegra.

En este sentido, las vacilaciones no se concentran en la cuestión de las vías, sino, en el modo de dar a conocer la fenomenología como tal. Por tanto, frente a la interpretación en términos de abandono del neocartesianismo, cabe afirmar que todas las modalidades son intentos de comprensión amplia pensadas como legado. Es por ello que lo que a primera vista puede dar la impresión de una variedad de proyectos sobrepuestos a la que hicimos referencia, constituye el resultado de la directriz teórica que se entronca con la idea originaria de fenomenología trascendental en su impulso más prístino de exploración de la fenomenicidad en todos sus aspectos. Esta misma línea está tan presente en los intentos tempranos, preocupados por manifestar aspectos nodales de la fenomenología, como en las exploraciones del último período, tal como se ve en el llamamiento a articular los distintos campos del enfoque sin provocar fracturas innecesarias en Meditaciones Cartesianas, en el $\S 58$ dedicado a la articulación de los problemas de la analítica trascendental de las comunidades intersubjetivas de orden superior, ${ }^{74}$ y se replica en los trabajos producidos entre 1930 y 1937 que conforman los manuscritos A sobre fenomenología mundana, los C sobre constitución del tiempo como constitución formal y los E sobre constitución intersubjetiva. En algún sentido, podría decirse que la multiplicación aumenta la potencia de una herencia en sí misma atravesada de dones para las generaciones de futuros fenomenólogos que desplegarían el entramado de las apropiaciones y despliegues de las distintas caras que Husserl fue poniendo a la fenomenología en repetidos intentos por alcanzar una fundamentación radical. 


\section{Bibliografía}

Adrián, J. (2005). "Hermeneutische versus reflexive Phänomenologie. Eine kritische Revision Heideggers früher Stellung zu Heidegger ausgehend vom Kriesgnotsemester 1919". Analecta Husserliana 88, pp. 157-173.

Adrián, J. (2011). "El joven Heidegger y los postulados de los prepuestos metodológicos de la fenomenología hermenéutica". Thémata. Revista de Filosofía 44, pp. 212-238. Ariew, R. (1999). Descartes and the last scholastics. Ithaca: Cornell University Press. Bernet, R., Kern, I., Marbach, E. (1995). An Introduction to Husserlian Phenomenology, Evanston: Northwestern University Press

Boehm, R. (1959) Erste phiiosophie II. Theorie der phaenomenologischen reduktion. The Halle, Martinus Nijhoff.

Braund, M. (2012) "From Inference to Empathy: Objectivity and Alterity in Husserl's Fifth Meditation", ms.

Bruzina, R. (1995) “Translator's introduction”, en E. Fink, Sixth Cartesian Meditation, Indiana: Bloomington.

Cairns, D. (1976) Conversations with Husserl and Fink. The Hague: M. Nijhoff. Carr, D. (1987) Interpreting Husserl: Critical and Comparative Studies. Dordrecht: Martinus Nijhoff.

Drummond, J. (1975) “The Structure of Intentionality”, en R. Bernet, D. Welton y G. Zavota (eds.) Critical Assessments of Leading Philosophers. London: Routledge, vol. 3. Gadamer, H. (1963) Wahrheit und Methode. Tubinga: Mohr.

Galt Crowell, A. (1990) "Husserl, Heidegger, and transcendental philosophy: Another look at the encyclopaedia britannica article". Philosophy and Phenomenological Research, 50, pp. 501-518.

García, E. (2012) Merleau-Ponty: Filosofía, corporalidad y percepción, Buenos Aires: Rhesis.

Geniusas, S. (2012). The Origins of the Horizon in Husserl's Phenomenology. Dordrecht: Springer.

Gurwitsch, A. (1942). Review of James Street Fulton, The Cartesianism of Phenomenology. Philosophy and Phenomenological Research, 2, 551-557.

Hermann, F. (1981) Der Begriff bei Husserl und Heidegger. Frankfurt: Klostermann. Hermann, F. (1990) Wege und Methode. Zur hermeneustischen Phänomenologie bei Heidegger und Husserl. Frankfurt: Klostermann.

Husserl, E.

Inverso, H. (2015) El mundo entre paréntesis. Una arqueología de las nociones de reducción y corporalidad. Buenos Aires: Prometeo.

Inverso, H. (2016a) Fenomenología de lo inaparente. Buenos Aires: Universidad de Buenos Aires -Tesis doctoral.

Inverso, H. (2016b) "La fenomenología de lo inaparente y el problema de las vías hacia el plano trascendental". Eidos, 26, pp. 43-73.

Inverso, H. (2017) “¿Qué es la fenomenología? La vía psicológica y la colaboración entre Husserl y Heidegger en el artículo de la Enciclopedia Británica”. Daimon, 70. Kockelmans, J. (1978) Edmund Husserl's Phenomenological Psychology: A Historico-Critical Study. Atlantic Highlands: Humanities Press. 
Landgrebe, L. (1970/2004) "Husserl's departure from Cartesianism" from Moran, D. - Embree, L.E., Phenomenology: Critical concepts in philosophy 5: Heritage of phenomenology. London: Routledge.

Luft, S. (2004) "Husserl's Theory of the Phenomenological Reduction: Between LifeWorld and Cartesianism". Research in Phenomenology, 34.

Mancosu, P. (2007), "Descartes and Mathematics", en J. Broughton et a. (Eds) A Companion to Descartes. Malden, MA: Blackwell, pp. 103-123.

Miettinen, T. (2013) The Idea of Europe in Husserl's Phenomenology : A Study in Generativity and Historicity. PHD Thesis.

Moran 2012 Husserl's Crisis of the European Sciences and Transcendental Philosophy. An Introduction. Cambridge: CUP.

Ricoeur, P. (1967) Husserl: An Analysis of His Phenomenology, trad. E.G. Ballard y L.E. Embree, Evanston, Northwestern University Press.

Rizzacasa, A. (1979) The Epistemology of the Sciences of nature in Relation to the Teleology of Research in the Thoght of the Later Husserl". Analecta Husserliana, 9. Russell, B. (1948) Human Knowledge: Its Scope and Limits. London: Allen.

Sánchez Soberano, E. (2012) E. Husserl, Las conferencias de Londres. Método y filosofía fenomenológicos. Madrid: Sígueme.

Sandmeyer, B. (2009) Husserl's Constitutive Phenomenology: Its Problem and Promise. New York: Routledge.

Sasaki, C. (2013) Descartes's Mathematical Thought. Dordrecht: Springer.

Schumann, K. (1977) Husserl-Chronik, Denk- und Lebensweg Edmund Husserls. La Haya: Martinus Nijhoff.

Scribano, E. (2016) "Descartes on Error and Madness". Rivista di Storia della Filosofia, 4.

Sheeham, T. - Palmer, R. (1997) Psychological and Transcendental Phenomenology and the Confrontation with Heidegger (1927-1931): The Encyclopaedia Britannica Article, The Amsterdam Lectures, "Phenomenology and Anthropology". Dordrecht: Springer

Staiti, A. (2012) "The Pedagogic Impulse of Husserl's Ways into Transcendental Phenomenology". Graduate Faculty Philosophy Journal, 33.1.

Steinbock, A. (1995) Home and Beyond: Generative Phenomenology After Husserl. Illinois: Northwestern University Press.

Steinbock, A. (1997) “Generativity and generative phenomenology”. Husserl Studies, 12.

Steinbock, A. (1998) "Husserl's Static and Genetic Phenomenology: Translator's Introduction to two Essays". Continental Philosophy Review, 31.

Steinbock, A. (2003) "Generativity and the Scope of Generative Phenomenology", en D. Welton, The New Husserl: A Critical Reader. Indiana: Indiana University Press.

Street Fulton, J. (1940) The Cartesianism of Phenomenology, Philosophical Review, (49), 285-308.

Tymieniecka, A. (2012) The Teleologies in Husserlian Phenomenology. Analecta Husserliana IX. Dordrecht: Reidel.

Velasco Guzmán, L. (2010) "Cartesian Metaphysics. The Late Scholastic Origins of Modern Philosophy". Multidisciplina, 6.

Walton, R. (2001) "Fenomenología de la empatía". Philosophica, 24. 
Zahavi, D. (2002) “Merleau-Ponty on Husserl: a Reapraisal”, en Toadvine, T., Embree, L. (eds.), Merleau-Ponty's Reading of Husserl. London: Springer.

Zahavi, D. (2005) Subjectivity and Selfhood: Investigating the First Person Perspective. Cambridge, MA: MIT Press. 